\title{
Creatividad y nuevas tecnologías: Las claves de la cultura emprendedora. El papel de la universidad en la formación de intelectuales con espíritu emprendedor
}

\author{
Luis Rodrigo Martín \\ Universidad de Valladolid \\ Francisco García García \\ Universidad Complutense \\ Isabel Rodrigo Martín \\ Universidad de Valladolid
}

\section{Palabras clave}

Comunicación; Universidad; Creatividad; Cultura emprendedora; Competencias; Nuevas tecnologías

\section{Resumen}

Vivimos en una sociedad azotada por una fuerte depresión económica y, lo que es más preocupante, una crisis de ideas y valores. Las respuestas tomadas desde los poderes públicos a esta situación, como recortes o contención, no han servido para resolver los problemas sino para poner en jaque la idea de un estado del bienestar, de naturaleza social, que trata de mitigar las desigualdades entre sus ciudadanos. Precisamente por este motivo se hace necesario buscar una solución más compleja y a largo plazo. Una estrategia que apueste de forma decidida y sin complejos por la educación, la investigación y la creatividad como ejes vertebradores de las diferentes políticas de recuperación. Una nueva universidad que tenga como fin principal formar ciudadanos con competencias intra e interpersonales, partícipes de una cultura emprendedora que permita dar respuestas a los problemas actuales y a los nuevos que se presentarán en el futuro. Nuevos modelos que generen ideas creativas e innovadoras encaminadas a la creación de empleo de calidad, incrementen el bienestar de los ciudadanos, ampliando sus espacios de participación activa, de comunicación y expresión, y construyendo una sociedad más equilibrada, presidida por la idea de justicia social, donde cobren importancia los valores del compromiso emocional, racional y ético, y que gestione la diversidad como una fuente de riqueza. 
Creativity and New Technologies: The keys to entrepreneurial culture. The role of universities in shaping intellectuals entrepreneurship

\title{
Keywords
}

Communication; University; Creativity; Entrepreneurial Culture; Skills; New Technologies

\begin{abstract}
We live in a society plagued by a severe economic depression and, more worryingly, a crisis of ideas and values. Answers taken from the public authorities to this situation, as cuts or containment, have failed to solve the problems but to jeopardize the idea of a welfare state, social nature, which seeks to mitigate inequalities among its citizens. Precisely for this reason it is necessary to seek a more complex and long-term solution. A strategy to bet decisively without complex for education, research and creativity as backbones of the different recovery policies. A new university that has as main purpose to educate citizens intra and interpersonal skills, participants in an entrepreneurial culture that allows to respond to current and new problems that arise in the future. New models that generate creative and innovative aimed at creating quality employment ideas, increase the welfare of citizens, expanding their areas of active participation, communication and expression, and building a more balanced society, dominated by the idea of social justice where gain importance values emotional, rational and ethical commitment, and to manage diversity as a source of wealth.
\end{abstract}

\section{Autores}

Luis Rodrigo Martín [lrodrigo@hmca.uva.es] es licenciado en Publicidad y RR.PP. y Derecho por la Universidad Complutense. Doctor en Comunicación Audiovisual con Premio Extraordinario de Doctorado por la Universidad de Valladolid. Es profesor de las asignaturas Procesos de creación del mensaje publicitario: medios y soportes y Gestión de Marca, ambas de la Universidad de Valladolid. Es también coordinador del Grupo de Investigación Tendencias en Publicidad y Consumo.

Francisco García García [fghenche@gmail.com] es catedrático de Comunicación Audiovisual y Publicidad de la Universidad Complutense de Madrid. Es director de las revistas académicas Icono 14, Creatividad y Sociedad y Prisma Social. Preside la Asociación Científica Icono 14. Es autor de numerosos libros y artículos en revistas académicas. 
Isabel Rodrigo Martín [isabel.rodrigo@uva.es] es licenciada en Publicidad y Relaciones Públicas en 2006 y doctora en Comunicación Audiovisual y Publicidad con Premio Extraordinario de Doctorado en 2012 por la Universidad de Valladolid. Es profesora de la Facultad de Ciencias Sociales, Jurídicas y de la Comunicación de Segovia (UVA) impartiendo Producción y Realización Publicitaria y Laboratorio Multimedia. Es también miembro de la Asociación Científica Icono 14. 


\section{Los retos de la Universidad}

En el inicio del siglo XXI se han producido una serie de acontecimientos de carácter geopolítico, social y económico que han propiciado un enorme cambio en la concepción del mundo y en la vida de sus ciudadanos. Como no podría ser de otra forma, esos cambios también han producido desajustes en los sistemas educativos, y particularmente en el papel de la Universidad como la institución encargada de la formación superior, garante de la investigación, del progreso y motor de la transformación social mediante el acceso al conocimiento. La Universidad necesita un cambio profundo para poder cumplir con su función de formar ciudadanos auténticamente capaces de poner sus competencias al servicio de la colectividad.

Desde esta perspectiva se hace precisa una intensa reflexión y un replanteamiento del papel de la universidad, ya que debe transformar la realidad y dar respuestas a las nuevas necesidades sociales que se están generando en una sociedad sumida en una profunda crisis.

De las crisis surgen las oportunidades y este podría ser el momento de que la comunidad científica, académica, artística e intelectual lidere un proyecto universitario ilusionante que permita, no solo superar la crisis económica, sino también abrir nuevos horizontes y oportunidades de desarrollo global y sostenible, que amplíen progresivamente el número de personas que pueden acceder a los bienes y servicios.

En realidad, el desafío no es nuevo, la universidad lleva siglos dando respuesta a las necesidades propias de cada momento histórico y de sus particularidades sociológicas. En el momento actual, desde nuestro punto de vista, la institución debe liderar el cambio formando a los nuevos ciudadanos en la cultura del emprendimiento e integrando las nuevas tecnologías en las rutinas de formación y educación. Si la universidad consigue dar respuesta a este desafío se reducirán considerablemente los dogmatismos, extremismos y fanatismos, ya que nada podrá ser considerado como indiscutible, por lo que existirán distintas verdades y diferentes realidades. La universidad tiene el poder de vencer la apatía y el conformismo proponiendo ideas innovadoras y acciones creativas que se constituyen en punto de inflexión para la evolución social, al mismo tiempo, deberá formar a ciudadanos creativos, que dominan las tecnologías del momento y dispuestos a innovar y a transformar mediante esa innovación. Todo ello presidido por una capacidad de cooperación que inspire los procesos transformadores en busca de alcanzar un interés común.

Tomando estas ideas como punto de partida identificamos la creatividad y las nuevas tecnologías como los pilares sobre los que debería asentarse la universidad de nuestros días. Un modelo universitario que pretendería el desarrollo de las competencias básicas del alumnado para que le permitan el desarrollo de la cultura del emprendimiento, y que gráficamente podríamos explicar de la siguiente manera: 


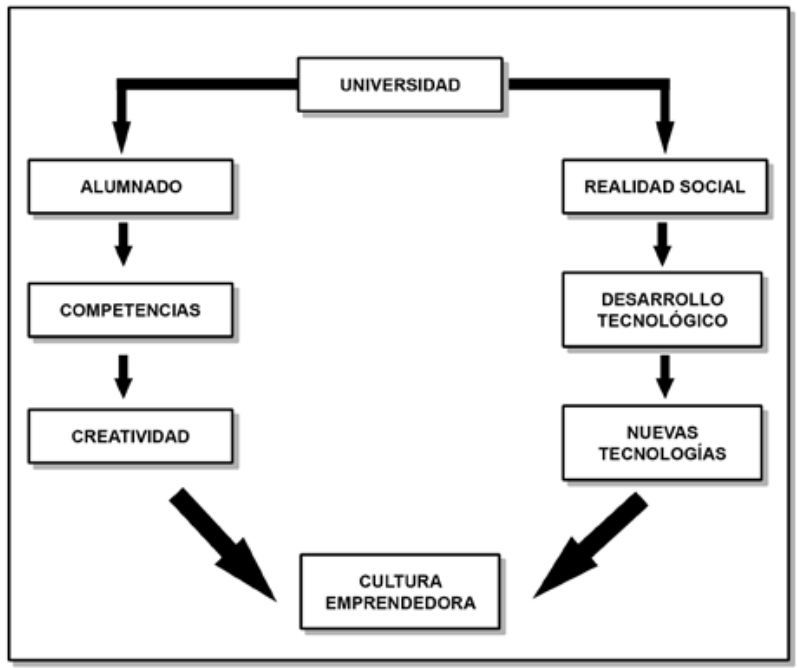

Elaboración propia

En la actualidad la tarea docente está recibiendo un nuevo estímulo que, al mismo tiempo, supone un importante desafío: la necesidad de afrontar los aspectos sociales del proceso formativo, a través de lo que se vienen denominando las competencias. A su vez, toma especial importancia la relevancia que están empezando a tener los aspectos afectivos y emocionales como anclaje del conjunto de actitudes, valores y normas que se precisa adquirir para poder llegar a una formación integral en la que no se prime la instrucción, como modelo de adquisición de conocimiento y de información, sino que se centre en el desarrollo de las competencias básicas, que capaciten al alumnado para aprender a aprender y enfrentarse a la realidad sabiendo, no sólo resolver los problemas que le presenta la realidad, sino detectando e identificando esos problemas y generando soluciones en un entorno dinámico y cambiante como es el mundo que ahora vivimos.

Así, el concepto de competencia, contrariamente a la interpretación de algunos autores, tiene en sí mismo un valor instrumental, en cuanto que se acerca a la formación integral en el que destaca el desarrollo de la personalidad y la habilidad para generar conocimiento y dominar las habilidades.

El concepto de competencia tiene una sustancia psicológica y social, sin embargo, ha saltado de la psicología a la educación y se está convirtiendo en un valioso instrumento creativo de cambio. 
El sentido de este término señala las habilidades personales y la capacidad para pensar, hacer y modificar nuestros comportamientos y las condiciones que nos rodean, siempre en un sentido positivo y de progreso social.

En definitiva, parece que se ha encontrado lo que siempre se ha estado buscando en la educación en general y en el sistema universitario en particular: hacer a los alumnos más competentes en todos los dominios del saber y del saber hacer, desde el pensamiento hasta la acción, sin olvidar los valores morales y éticos necesarios en toda convivencia.

\section{Creatividad}

El concepto creatividad es, hoy más que nunca, un tema de actualidad en diferentes ámbitos de conocimiento por la importancia que tiene, tanto en el desarrollo personal como en la capacitación y éxito profesional, en una sociedad tan compleja como la nuestra que obliga a adecuarse permanentemente a las diferentes y difíciles demandas que surgen en su seno.

Las investigaciones realizadas desde el campo de la psicología sobre creatividad se han centrado principalmente en dos procesos: la inteligencia y la personalidad (Keith, 2000). La relación entre inteligencia y creatividad aparece desde los primeros estudios: Galton (1879), que concluía que los productos creativos procedían de una habilidad general; W. James (1880) que formula la idea de pensamiento divergente; Binet y Spearman (1904) que incluyen en su escala de inteligencia ítems que evaluaran la imaginación; y Terman y Cox (1926) que apuntan que la creatividad es una parte integral de la inteligencia. Guilford en 1950 apuesta por emprender una investigación empírica sobre la creatividad y, partiendo de sus estudios sobre la inteligencia, entiende este constructo como la combinación del pensamiento convergente y divergente. Es, precisamente a partir de las aportaciones de este autor, cuando la identidad de la creatividad iniciará su verdadero desarrollo en el campo científico (Castejón, Prieto y López, 2000). Las conclusiones de estos primeros estudios ratifican que existe una clara relación entre inteligencia y creatividad pero que se requiere de un alto C.I. para llegar a ser creativo (Keith, 2000).

Los avances en la investigación sobre creatividad han sido permanentes y debemos destacar las aportaciones de Gardner (1990) quien demuestra que existen múltiples inteligencias, y que se puede puntuar alto en algunas de ellas y no en otras, por lo que su relación con la creatividad vendrá dada más por el tipo de inteligencia medida que por un constructo más abstracto y amplio al que hemos llamado inteligencia.

Si nos fijamos en el otro concepto objeto de estudio, la personalidad y su relación con la creatividad, encontramos estudios que asocian la creatividad a ciertas patologías que padecen las personas creativas. Romo (1998) a través de la teoría del trastorno psicológico, la teoría de la búsqueda de sí mismo y la teo- 
ría de la expresión emocional. Otras concepciones más psicológicas apuntaban que la persona considerada creativa, estaba dotada de determinadas patologías o rasgos de personalidad, entre los que destacaban principalmente: tendencia al consumo de sustancias adictivas y una introversión extremada con cuadros de extraversión descontrolada. Estas características, se decía, permitían a los creadores ser poseedores de esa genialidad (Fuentes Ramos y Torbay Betancor, 2004).

Investigaciones posteriores rebaten estas teorías y demuestran que las personas creativas no tienen que presentar necesariamente rasgos patológicos en su personalidad. Ahora bien, podemos concluir que sí parecen haber demostrado que las personas creativas poseen determinados rasgos, aunque estos no tienen nada de patológicos (Keith, 2000).

Por tanto, si consideramos la creatividad entre los procesos, inteligencia y personalidad, estamos considerando a la creatividad como una característica inherente al ser humano; es decir, la creatividad es una capacidad que poseen todas las personas y que es susceptible ser desarrollada con el esfuerzo y el entrenamiento.

Los avances en las investigaciones han señalado que la creatividad no se encuentra sólo en la mente de los sujetos, ni depende exclusivamente de factores cognitivos. Sentimientos, emociones, motivaciones, representaciones, percepciones, historias de vida, interacciones sociales, contextos particulares, entre otros factores, serían determinantes de las posibilidades de desplegar la creatividad en diversas situaciones.

Son muchas las orientaciones que abordan el fenómeno de la creatividad desde las diferentes escuelas o tendencias, produciendo resultados muy diferentes $\mathrm{y}$, en muchos casos, imposibles de integrar en una única teoría global y comprensiva, pero todas estas aportaciones convergen en la formulación de los cuatro aspectos fundamentales concernientes a la creatividad o la denominación de Isaksen, Dorval y Treffinger (2000) de «las cuatro pes de la creatividad»: Persona, Producto, Proceso y Presión-Contexto (Press, referido en español a contexto):

- Persona, en cuanto a sus características diferenciales como creativo.

- Proceso, por el cual se obtienen resultados creativos.

- Producto, creativo.

- Presión-Contexto, que hace posible las conductas creativas.

Encontramos por tanto un consenso científico al considerar en la actualidad a la creatividad como una competencia del individuo que se desarrollará en gran medida, según la apropiación y la reconstrucción de las posibilidades y oportunidades que ofrecen los distintos contextos en los que vivimos. 
En este sentido, autores como Guidano y Quiñones (2001) exponen que la creatividad ya no puede ser vista únicamente como potencialidad individual, solo para el sujeto, sino que debe considerarse al sujeto como un ser social que interactúa con la realidad, con el contexto o situación en que se desenvuelve, y con todas las demandas sociales, educativas, empresariales, laborales, etc., que presentan las sociedades en un momento determinado.

Por estas razones, consideramos las dos vertientes de la creatividad, una individual y otra social, porque la creatividad no sólo tiene sentido para y por el desarrollo personal, sino que las diferentes formas que tiene crear e innovar se entienden al servicio del desarrollo individual y también del desarrollo y progreso de la sociedad.

La universidad debe tener como principio fundamental el desarrollo creativo de su alumnado, de esta forma se podrá ir construyendo una cultura emprendedora donde los alumnos aprendan desde las aulas el desarrollo de su autonomía e independencia, la asunción de riesgos para llegar a descubrimientos importantes, la autoconfianza en sus capacidades y el autocontrol de sus conductas y emociones, rasgos que definen a las personas emprendedoras.

Esta es la razón por la que la creatividad ha de estar presente, de forma transversal, en todas y cada una de las actividades. No podemos encasillarla en un horario como una disciplina más a la que se dedica unas horas semanales. La creatividad debe llenar la vida de la universidad y debería convertirse en el estilo docente. Cualquier situación universitaria es buena para reflexionar con el alumnado. Consideramos que no se deben dejar escapar las ocasiones donde aparece la admiración, el interrogante, la inquietud, y que deberían aprovecharse las preguntas y las infinitas curiosidades de los alumnos para ayudarles a buscar, sentir, crear y emprender.

La necesidad de fomentar y aumentar la presencia de la creatividad en el seno de la universidad no debe articularse de una manera meramente formal, no se trata de introducir o incorporar una nueva disciplina, sino de una forma mucho más sistémica, mediante la rentabilización muchas de las actividades que se realizan de forma cotidiana en las aulas universitarias. La selección de algunas de las habilidades debería formar la urdimbre del contenido formativo, la trama se debería crear a partir de las actividades. Cada habilidad sería convenientemente explicitada y los ejercicios que se planteen serán testados en las aulas.

\section{Las nuevas tecnologías de la información y del conocimiento}

Vivimos en una sociedad donde el desarrollo tecnológico ha transformado significativamente las formas de recoger información, de relacionarnos y de trabajar. La comprensión y el correcto manejo de las nuevas posibilidades tecnológicas se convierten en requisito imprescindible para estar integrado y formar parte 
de las clases sociales que tienen la posibilidad de pertenecer a los ámbitos de representación y de poder.

Como casi todo lo que supone una novedad y obliga a un cierto esfuerzo de adaptación, las nuevas tecnologías de la información han sido criticadas desde algunos sectores, en la mayor parte de los casos por desconocimiento o recelo ante las nuevas posibilidades que permiten y la universidad no ha sido una excepción.

Al hablar de las nuevas tecnologías aplicadas a la formación universitaria debemos señalar que por lo general suele ser el uso que se haga de ellas, y no sus características específicas, lo que determina las consecuencias positivas o negativas de su aplicación.

Así, podemos señalar como ventajas:

- Variedad metodológica y atención a la diversidad del alumnado.

La existencia de diferentes estilos de aprendizaje y diferentes modos de enseñar aconsejan la conveniencia de una educación multimedia.

- Facilitan el tratamiento, la presentación y la comprensión de ciertos tipos de información.

En este momento sabemos que existe la posibilidad de digitalizar la información verbal y audiovisual como una de las características de las nuevas tecnologías. Esto permite un gran control sobre la información para su clasificación, búsqueda, transmisión, etcétera. Los sistemas multimedia pueden llevar a cabo el tratamiento de la información mejor que los seres humanos, y complementar la superior capacidad cognitiva que, por contra, poseen las personas.

- Facilitan el protagonismo del alumno en su propio aprendizaje.

Esta característica está directamente relacionada con la interactividad propia de las nuevas tecnologías que permiten al alumno relacionarse con el sistema.

Entre los posibles inconvenientes, podemos señalar:

- Percepción del medio como fácil. Pasividad.

El aprendizaje no se produce por el mero contacto con el medio y sus documentos sino a través de una interacción reflexiva y consciente con sus contenidos. Para ello, es necesaria una predisposición adecuada por parte de alumno y profesor. Tanto el uno como el otro, que son consumidores habituales de productos audiovisuales y multimedia, acuden a las clases con hábitos de visionado doméstico de pasividad y falta de atención, inadecuados para la enseñanza-aprendizaje con tecnologías, lo que constituye un posible inconveniente en la utilización didáctica de estas tecnologías.

- Uso inadecuado y abuso 
Al tratar anteriormente las posibles razones de incorporación de las nuevas tecnologías a la enseñanza y la posturas del profesorado advertíamos del peligro que supone partir de la inevitable incorporación y buscar después cualquier tipo de aplicaciones didácticas que la justifiquen. Esta postura genera dos tipos de problemas: el abuso, o la utilización excesiva, y el uso inadecuado, consistente en la utilización de un medio cuando existe otro más efectivo y de más fácil utilización.

En muchos casos, la incorporación de un medio a las situaciones docentes no obedece a la especificidad de ese particular medio para favorecer el aprendizaje, sino en la necesidad del profesor de estar al día, o en el atractivo que supone para los alumnos. Aunque estas pudieran ser dos razones válidas para plantearse la integración de las nuevas tecnologías, no lo son directamente para su integración. La integración curricular, a diferencia de la incorporación, supone que el uso de un medio es el resultado del análisis conjunto de todos los elementos del currículo, y que, la elección de un dispositivo o un modo de presentar la información, viene determinada por el tipo de objetivos y contenidos.

El uso inadecuado de las nuevas tecnologías suele estar motivado por: incoherencia con los contenidos o con las funciones que se les asignan.

La superabundancia de información en los documentos multimedia, y en redes de comunicación como Internet, puede resultar en inconveniente, si ni profesores ni alumnos dominan las destrezas de acceso y selección de tan gran cantidad de datos. Para aprender a seleccionar la información y adaptarla a los intereses de cada situación particular, para triunfar en la era de la información, considera Wilson (1991) más necesario que nunca el desarrollo de destrezas interpretativas, como la resolución de problemas, el pensamiento creativo y una actitud de búsqueda y apertura.

\section{Universidad y espíritu emprendedor}

El espíritu emprendedor comienza a formarse en el colegio. Con frecuencia se citan cifras al respecto extraídas de las encuestas realizadas dentro de los países de la Unión Europea. «Entre el 15 \% y el 20 \% de los escolares que participan en programas educativos de creación de pequeñas compañías serán empresarios más tarde. Eso está, entre tres y cinco veces, por encima de la media y demuestra la importancia del sistema educativo en el desarrollo de las actitudes emprendedoras» (Programa Integral para el Fomento de la Cultura Emprendedora 2013-2015. Principado de Asturias: 23).

La recomendación de las políticas europeas es clara en este sentido, se anima a los estados miembros de Unión Europea (UE) a que incluyan en los sistemas de enseñanza obligatoria programas para promover el espíritu emprendedor, siguiendo directrices marcadas por la propia OCDE. La educación, por tanto, es fundamental para transformar, desde abajo y por generaciones sucesivas, la 
aversión al riesgo y, por contra, potenciar el espíritu emprendedor, de cambio, de actitud hacia una nueva transformación de lo que supone el empleo tal y como se conoce en la actualidad.

A principios de enero de 2013, la Comisión Europea (CE) presentó el Plan de Acción sobre el Emprendimiento 2020 (3), con la finalidad de «relanzar el espíritu emprendedor en Europa». Un plan que arranca con unos acotamientos muy claros a los que Europa tiene que hacer frente y, si cabe, mucho más en España y en una economía como la española. Por ello, merece la pena resaltar algunos de los datos y estrategias más importantes que se señalan desde la propia CE y que son claves para entender la importancia de una educación que fomente el desarrollo del emprendimiento.

La propia Comisión indica que «el emprendimiento es un importante motor de crecimiento económico y creación de empleo: crea nuevas empresas y empleos, abre nuevos mercados y favorece nuevas competencias y capacidades. (...)»

El emprendimiento hace que la economía sea más competitiva e innovadora, y es fundamental para alcanzar objetivos como:

- Comercializar nuevas ideas mejora la productividad y crea riqueza. Sin los puestos de trabajo creados por nuevas empresas, el crecimiento medio neto del empleo sería negativo.

- Las nuevas empresas, y especialmente las PYME, representan la fuente más importante de nuevos empleos: crean más de cuatro millones de puestos de trabajo al año en Europa.

- Pero el motor de esa recuperación está fallando: desde 2004, la proporción de personas que prefieren trabajar por cuenta propia antes que convertirse en asalariadas ha disminuido en veintitrés de los veintisiete Estados miembros de la UE.

- Mientras que el trabajo por cuenta propia era hace tres años la primera opción del $45 \%$ de los europeos, este porcentaje ha bajado ahora hasta el $37 \%$.

- En cambio, en los Estados Unidos y China esta proporción es mucho mayor: el $51 \%$ y el $56 \%$ respectivamente.

Además, al crear nuevas empresas, estas crecen más lentamente en la UE que en los Estados Unidos o en países emergentes, y menos de ellas llegan a formar parte de las mayores empresas del mundo (Comisión Europea, 2013).

La universidad tiene el compromiso de plantear unos contenidos y metodologías que sirvan para el fomento de la cultura emprendedora. Su filosofía de actuación se basa en que, para conseguir una ciudadanía emprendedora, se deben poner las herramientas adecuadas para que ello se produzca. Por ello, la tarea a realizar debe comenzar en los centros educativos, a través de la integración en 
el currículo de materias y módulos relacionados con la cultura emprendedora y seguir trabajando en los niveles educativos superiores. Cuando se quiere trabajar en la educación emprendedora es imprescindible el desarrollo de perfiles emprendedores que contribuyan a un cambio social.

Esto se logra a través del trabajo personal de la competencia emprendedora referida a la capacidad de las personas de llevar las ideas a la acción.

«Emprender es cuestión de actitud, de espíritu, de creer en algo, de arriesgarse, de esforzarse, de no pararse ante los problemas, sino de intentar resolverlos. Y para forjar esa mentalidad es clave empezar desde abajo, desde la escuela, para que la mente se vaya amoldando a otra forma de ver la vida» (Calle, 2012: 3).

La universidad tiene que plantear desde los primeros cursos programas y proyectos educativos que capaciten al alumnado a desarrollar los rasgos que definen a las personas emprendedores.

Son muchos los estudios que tratan de definir los rasgos de las personas emprendedoras; en el estudio editado en el Libro Blanco de la iniciativa emprendedora en España (2011) se señalan los siguientes:

-Independencia

-Propensión al riesgo

-Autoconfianza

-Creatividad

-Autocontrol

No obstante, además, se necesita pasión, ilusión, perseverancia, sacrificio y buenas dosis de creatividad, y capacidad para diseñar un proyecto y sacarlo adelante. Es por tanto, un camino de superación continúo:

«Tienes que tener un evidente afán de superación constante y cierto espíritu aventurero» (Alonso, 2012: 29).

\section{Conclusiones}

Nos encontramos inmersos en un momento de crisis que posiblemente acabe con el estilo de vida que venimos disfrutando. En el momento actual, es complicado saber hacia dónde evolucionarán nuestros hábitos y costumbres pero, lo que sí que parece evidente, es que debemos de enfrentarnos a nuevos retos y desafíos. Algunos conceptos como el trabajo, las pensiones, nuestra relación con el espacio y el tiempo, la tecnología, la comunicación, etc. se enfrentan a una transformación radical. En este momento decisivo en el que se empieza a forjar el modelo de vida de las próximas épocas la universidad es una pieza imprescindible en la construcción del puzle social. Sin embargo, la institución universitaria también precisa un cambio fundamental para poder formar ciudadanos 
creativos, capaces de innovar y diferenciarse de la masa, con las habilidades suficientes para convivir de forma democrática y gratificante, lo que permitirá el desarrollo de las competencias básicas del individuo, el dominio de las tecnologías y la adquisición de una cultura del emprendimiento.

La universidad del siglo XXI debe fijarse como objetivos fundamentales formar ciudadanos con capacidad suficiente para romper la uniformidad y conseguir diferenciarse, sin miedo a innovar, capacitados para el trabajo en equipo y con valores como el esfuerzo, la tenacidad y el entusiasmo.

Para poder alcanzar estos objetivos se hace preciso una nueva metodología universitaria que deberá sustentarse en:

- La observación

- La experimentación

- El descubrimiento

- El reconocimiento

Y que fomente el aprendizaje desde la propia experiencia, apueste por la creatividad y la innovación, promoviendo el autodescubrimiento, generando preguntas en el alumnado antes que ofrecer respuestas de manual, que promueva la investigación, la deducción y la capacidad crítica para confrontar saberes.

En definitiva, una universidad que dote al individuo de los recursos necesarios para la toma de decisiones al tiempo que anima a la puesta en práctica de las mismas, erradicando el miedo al fracaso y construyendo ciudadanos capacitados, cualificados y emprendedores.

\section{Bibliografía}

Alonso, A. (2012) citado por Bustos, V. Así son sus genes. En: Capital, número extraordinario. Madrid: Dixi Media publicidad.

Calle, C. (2012). Pasión y perseverancia. En: Capital, número especial. Madrid: Dixi Press.

Cantillon, R. (1756). Essai sur la Nature du Commerce en General. Londres y París: R. Gyles.

Castejón, J. L.; Prieto, M. D. y López, O. (2000). Evaluación y desarrollo de la creatividad. En: Prieto, M. D. y Castejón, J. L. (eds). Los Superdotados. Esos alumnos excepcionales. Málaga: Ed. Aljibe.

Comisión de las Comunidades Europeas (2003). Libro verde. El espíritu empresarial en Europa. Bruselas. 
Comisión Europea (2004). Implementation of "Education and Training 2010. Work programme. Directorate General for Education and Culture.

Comisión Europea (2013). Plan de acción sobre emprendimiento 2020. Relanzar el espíritu emprendedor en Europa. Bruselas, 9.1.2013 . COM(2012). Disponible en: «http://www.emprenderencanarias.es/wp-content/uploads/2013/03/ Plan-de-Accio\%CC\%81n-sobre-Emprendimiento-2020-Europa.pdf» (Consultado: 25/11/2014).

Cruz, C. (2008). Fuentes de financiación y actividad emprendedora: España en el contexto internacional. En: De Castro, J.O.; Justo, R. y Maydeu, A. (eds.). La naturaleza del proceso emprendedor en España en el contexto internacional. Madrid: Fundación BBVA, 157-189.

Cuadrado Alvarado, A (2011). Utopías y Distopías de los medios digitales en la Educación. En: Icono 14. Revista de comunicación y de tecnologías emergentes, vol. $9, \mathrm{n}^{\circ} 2,5-20$.

Fuentes Ramos, R.; Torbay Betancor, A. (2004). Desarrollar la creatividad desde los contextos educativos: un marco de reflexión sobre la mejora sociopersonal. En: REICE. Revista Iberoamericana sobre Calidad, Eficacia y Cambio en Educación, vol. 2, núm. 1, enero-junio.

Gardner, R. (1990). When children and adults do not use learning strategies: toward a theory of settings. En: Review of Educational Research, vol. 60, $\mathrm{n}^{\mathrm{0}} 4$, 517-529.

Gartner, W.B. (1985). A conceptual framework for describing the phenomenon of new venture creation”. En: Academy of Management Review, vol. 10, $n^{0} 4$, 696-706.

Guidano, V. F.; Quiñones, A. (2001). El modelo cognitivo postracionalista. Hacia una reconceptualización teórica y crítica. Bilbao: Editorial Descleé de Brouwer, S. A.

Hernández, N; González, M y Muñoz, P. (2014). La planificación del aprendizaje colaborativo en entornos virtuales. En: Revista Comunicar. Revista científica de comunicación y educación, $\mathrm{n}^{0} 42,25-33$.

Isaksen, S. G; Dorval, K. B. y Treffinger, J. D. (2000). Creative approaches to problem solving. Aframerwork for change. Williamsville. Nueva York: Group-Buffalo.

Keith, D. (2000). Cognitive, Personal, Developmental, and Social Aspects. En: American Psychologist, $\mathrm{n}^{0}$ 55, 151-158.

Prada, J. M. de (2012). Emprendedor. En: Capital. Número extraordinario. Madrid: Dixi Media publicidad. 
Rodrigo Martín, I., Rodrigo Martín, L. y Martín Requero, M. (2013). Enseñanza y aprendizaje de la creatividad en la educación formal. En: Revista Creatividad y Sociedad, $\mathrm{n}^{0} 21$.

Wilson, K. (1991). New tools for new learning opportunities. En: Revista Technology \& Learning, vol.11, $\mathrm{n}^{\mathrm{0}} 7,1991$.

\section{Referencia de este artículo}

Rodrigo Martín, Luis; García García, Francisco y Rodrigo Martín, Isabel (2014). Creatividad y nuevas tecnologías: Las claves de la cultura emprendedora. El papel de la universidad en la formación de intelectuales con espíritu emprendedor. En: adComunica. Revista Científica de Estrategias, Tendencias e Innovación en Comunicación, $\mathrm{n}^{\circ} 8$. Castellón: Asociación para el Desarrollo de la Comunicación adComunica, Universidad Complutense de Madrid y Universitat Jaume I, 23-37. DOI: http://dx.doi.org/10.6035/2174-0992.2014.8.3. 
